\title{
Shubnikov-de Haas Oscillations in Digital Magnetic Heterostructures
}

\author{
Henrique J. P. Freire and J. Carlos Egues \\ Departamento de Física e Informática, Instituto de Física de São Carlos, \\ Universidade de São Paulo, C.P.369, 13560-970 São Carlos-SP, Brazil
}

Received on 8 March, 2003

\begin{abstract}
In this paper we theoretically investigate the magnetic-field and temperature dependence of the Shubnikov-de Haas oscillations in group II-VI modulation-doped Digital Magnetic Heterostructures. We self-consistently solve the effective-mass Schrödinger equation within the Hartree approximation and calculate the electronic structure and the magneto-transport properties. Our results show $i$ ) a shift of the Shubnikov-de Haas minima to lower magnetic fields with increasing temperature, and $i$ ) an anomalous oscillation which develops when two opposite Landau levels cross near the Fermi energy. Both of these are consistent with recent magneto-transport measurements in such heterostructures [R. Knobel et al., Phys. Rev. B 65, 235327 (2002)].
\end{abstract}

\section{Introduction}

Digital Magnetic Heterostructures (DMHs) are semiconductor heterostructures where magnetic monolayers are incorporated using the digital-alloy technique $[1,2]$. The $s-d$ exchange interaction in such systems is responsible for a magnetic-field- and temperature-dependent giant spin splitting of the electronic bands [3], thus giving rise to strong spin-dependent effects. This giant splitting is up to two orders of magnitude larger than the ordinary Zeeman effect and is easily observed by magneto-photoluminescence [4]. The successful achievement of high doping carrier densities in such magnetic layered structures [5] has enabled magneto-transport and magneto-photoluminescence measurements in these systems $[1,5]$. More recently, the $s-d$ exchange enhanced spin-splitting has been used to align opposite spin Landau levels near the Fermi level [6, 7], revealing striking features in the electronic structure and magnetotransport properties of such two-dimensional electron systems.

In Ref. 6 magnetization and magneto-transport measurements in $n-\mathrm{ZnSe} /(\mathrm{Zn}, \mathrm{Cd}, \mathrm{Mn}) \mathrm{Se} \mathrm{DMHs}$ were performed in the quantum Hall regime for a wide range of temperatures and carrier densities. In this paper we focus our analysis on two particular features of the experimental results, namely $i$ ) a shift of the Shubnikov-de Haas $(\mathrm{SdH})$ peaks to lower magnetic fields as the temperature is raised and $i$ ) an anomalous peak in the $\rho_{x x}$ data near $B \sim 3.2$ T. We selfconsistently solve the effective-mass Schrödinger equation within the Hartree approximation. Our results reproduce the reported temperature dependence of the $\mathrm{SdH}$ peaks and the anomalous oscillation. In the following sections we present our model and results.

\section{Model and Approach}

The system we study consists of a modulation doped digital magnetic quantum well [6]. One $1 / 16$ monolayer of MnSe is inserted every 7 monolayers in a $105 \AA(\sim 35$ monolayers) wide $\mathrm{Zn}_{0.87} \mathrm{Cd}_{0.13}$ Se quantum well. The well is surrounded by two $120 \AA$ intrinsic ZnSe spacers, each followed by a $200 \AA n$-doped $\mathrm{ZnSe}$ layer. The measured low-field two-dimensional density is $n_{s}=2.8 \times 10^{11}$ $\mathrm{cm}^{-2}$. The strong Coulomb repulsion between confined electrons, which deforms the potential profile, is treated within the Hartree approximation. We self-consistently solve the effective-mass Schrödinger equation

$$
\left[-\frac{\hbar}{2 m} \frac{\partial}{\partial z^{2}}+v_{e f f}^{\sigma_{x}}(z ; B, T)\right] \chi_{i}^{\sigma_{z}}(z)=\varepsilon_{i}^{\sigma_{z}} \chi_{i}^{\sigma_{z}}(z),
$$

where $m=0.145 m_{0}$ is the electron effective mass, $i=$ $1,2, \ldots$ is the subband index, and $\sigma_{z}= \pm 1$ denotes the spin components. The effective potential is

$v_{e f f}^{\sigma_{x}}(z ; B, T)=v_{0}(z)+v_{b}(z)+v_{s-d}^{\sigma_{z}}(z ; B, T)+v_{h}(z)$,

where $v_{0}(z)$ is the the quantum well profile with band offset of $210 \mathrm{meV}$ [8]; $v_{b}(z)$ is the Mn barrier profile with an assumed height of $800 x_{p}$ meV [9], $x_{p}$ being the nominal Mn concentration; $v_{s-d}^{\sigma_{z}}(z ; B, T)$ is the $s$ - $d$ exchange contribution given by [10]

$$
v_{s-d}^{\sigma_{z}}(z ; B, T)=\frac{\sigma_{z}}{2} N_{0} \alpha x(z) \frac{5}{2} B_{5 / 2}\left[\frac{5 \mu_{B} B}{k_{B}\left(T+T_{0}\right)}\right],
$$

where $N_{0} \alpha=0.26 \mathrm{eV}$ is the $s$ - $d$ exchange constant, $x(z)$ is an effective Mn concentration profile $[11,12], B_{5 / 2}$ is the spin-5/2 Brillouin function, and $T+T_{0}$ is an effective temperature; $v_{h}(z)$ is the Hartree potential which is calculated 
by solving the Poisson equation. Each Landau level energy is then given by

$$
\varepsilon_{i, n}^{\sigma_{z}}=\varepsilon_{i}^{\sigma_{z}}+\left(n+\frac{1}{2}\right) \hbar \omega_{c}+\frac{\sigma_{z}}{2} g^{*} \mu_{B} B
$$

where $n$ is the Landau level index, $\hbar \omega_{c}$ is the cyclotron energy, and $g^{*}=0.4$ [13] is the effective Landé factor of the Zeeman term.

We use gaussian broadened Landau levels with a density of states (DOS) for each spin subband given by

$$
g_{i, \sigma_{z}}(\varepsilon)=\frac{e B}{h} \sum_{n} \frac{1}{\Gamma \sqrt{2 \pi}} \exp \left[-\frac{\left(\varepsilon-\varepsilon_{i, n, \sigma_{z}}\right)^{2}}{2 \Gamma^{2}}\right]
$$

where $\Gamma$ is the broadening width. Using this DOS together with the Fermi function $f(\varepsilon)=\left\{1+\exp \left[(\varepsilon-\mu) / k_{\mathrm{B}} T\right]\right\}^{-1}$ we self-consistently calculate the chemical potential by solving the $2 \mathrm{D}$ density

$$
n_{s}=\sum_{i, \sigma_{z}} \int_{-\infty}^{\infty} g_{i, \sigma_{z}}(\varepsilon) f(\varepsilon) \mathrm{d} \varepsilon
$$

for $\mu$.

We calculate the longitudinal conductivity using [14]

$$
\begin{aligned}
\sigma_{x x} & =\frac{e^{2}}{\hbar \pi^{2}} \int_{-\infty}^{\infty}-\left(\frac{\partial f(\varepsilon)}{\partial \varepsilon}\right) \\
& \times \sum_{i, n, \sigma_{z}}\left(n+\frac{1}{2}\right) \exp \left[-\left(\frac{\varepsilon-\varepsilon_{i, n, \sigma_{z}}}{\Gamma_{\mathrm{ext}}^{\sigma_{z}}}\right)^{2}\right] \mathrm{d} \varepsilon
\end{aligned}
$$

where $\Gamma_{\mathrm{ext}}^{\sigma_{z}}$ is the width of the extended states region within each $\Gamma$-broadened Landau level. The transversal conductivity is given by the Drude model $\sigma_{x y}=-e n_{s} / B$. To calculate the resistivities we simply invert the conductivity tensor $\rho=\sigma^{-1}$.

\section{Results}

The electronic structure and magneto-transport calculations were performed at four different temperatures $T$. We use $x_{p}=0.093$ and $n_{s}=2.8 \times 10^{11} \mathrm{~cm}^{-2}$ (the low field measured areal density [6]) to obtain a good agreement with the experimental findings. For the other parameters we use $T_{0}=1.55 \mathrm{~K}, \Gamma=0.36 B^{1 / 2} \mathrm{meVT}^{-1 / 2}, \Gamma_{\text {ext }}^{\downarrow}=0.25 \mathrm{meV}$, and $\Gamma_{\text {ext }}^{\uparrow}=0.05 \mathrm{meV}$, the same values as in Ref. 6 .

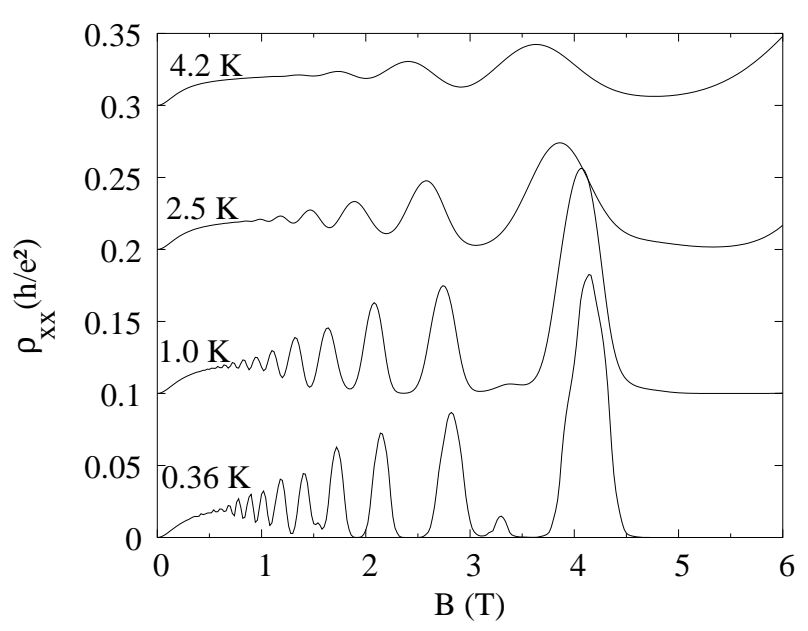

Figure 1. Calculated Shubnikov-de Haas oscillations for different temperatures. Oscillations in $\rho_{x x}$ shift to lower fields as $T$ increases. An anomalous peak develops at $B_{c} \sim 3.2 \mathrm{~T}$ for $T=0.36$ $\mathrm{K}$ and at $B_{c} \sim 3.4 \mathrm{~T}$ for $T=1.0 \mathrm{~K}$. The shift to lower fields of the $\mathrm{SdH}$ peak at $B \sim 4 \mathrm{~T}$ from $T=1.0 \mathrm{~K}$ to $T=2.5 \mathrm{~K}$ is bigger than from $T=0.36 \mathrm{~K}$ to $T=1.0 \mathrm{~K}$, in accordance to experiment [6]. The Fermi function softens the oscillations for higher temperatures, thus making them wider. The offset between curves is $0.1 \mathrm{~h} / \mathrm{e}^{2}$.

Figure 1 shows the theoretical magnetic field and temperature dependencies of $\rho_{x x}$. In accordance with the experimental results [6], our calculated $\mathrm{SdH}$ oscillations $i$ ) shift to lower magnetic fields as the temperature increases, and ii) show an anomalous peak at $B_{c} \sim 3.2 \mathrm{~T}$.

The temperature dependence of the Mn magnetization [Brillouin function in Eq. (3)] is responsible for the $\mathrm{SdH}$ shift, as pointed out in Ref. 6. As the temperature rises, the $s$ - $d$ enhanced spin splitting decreases and the spin down energy increases (Fig. 2), crossing the Fermi energy at a lower $B$ field. Note that temperature also plays a role via the Fermi distribution, which smooths the $\mathrm{SdH}$ oscillations as $T$ increases (Fig. 1). However, its effect on the SdH shift within the investigated temperature range is small when compared to the $s-d$ exchange contribution.

The anomalous peak that develops at $B_{c} \sim 3.2 \mathrm{~T}$ (Fig. 1, $T=0.36 \mathrm{~K}$ ) is a result of a Landau level crossing near the Fermi energy [Fig. 2(a)]. At $T=0.36 \mathrm{~K}$, just below $B_{c}$, a $\mathrm{SdH}$ oscillation takes place while the Fermi energy crosses the $|3, \downarrow\rangle$ level. Meanwhile the $|0, \uparrow\rangle$ is increasing in energy and becomes degenerate with $|3, \downarrow\rangle$. As the magnetic field increases, the Fermi energy crosses the spin up level just before it becomes completely empty, resulting in the small $\mathrm{SdH}$ peak. This effect is suppressed for higher temperatures as the Fermi function increases the overlap between levels thus making them indistinguishable and giving rise to the wider peaks at $T=2.5 \mathrm{~K}$ and $T=4.2 \mathrm{~K}$ in Fig. 1 . 


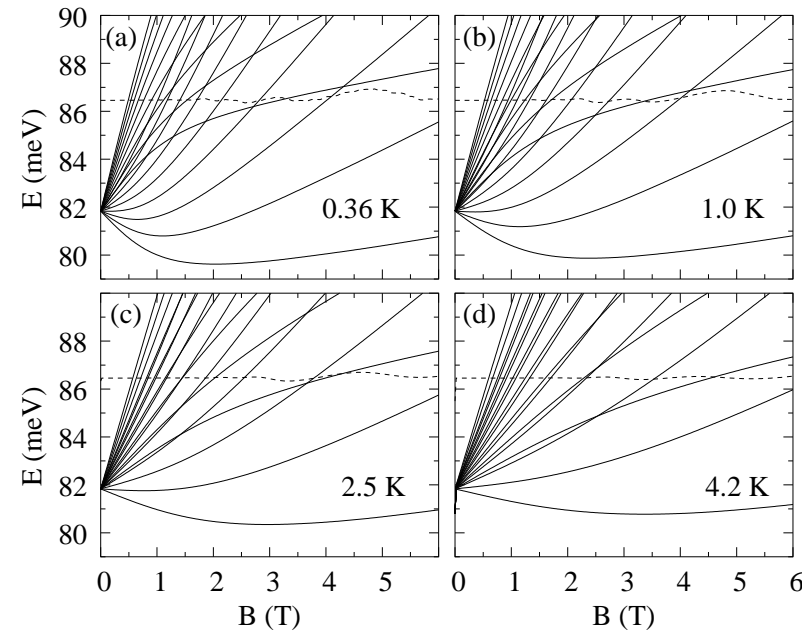

Figure 2. Landau level fan diagrams for different temperatures. In (a) and (b) the Fermi level crosses the $|3, \downarrow\rangle$ Landau level just after the crossing of $|3, \downarrow\rangle$ and $|0, \uparrow\rangle$, thus giving rise to an extra Shubnikov-de Haas oscillation shown in Fig. 1. At $T=2.5 \mathrm{~K}$ (c) the $|2, \downarrow\rangle$ and $|0, \uparrow\rangle$ levels cross at $B \sim 3.7 \mathrm{~T}$ near the Fermi energy, thus originating a wide oscillation shifted to lower $B$ fields.

\section{Discussions and Conclusions}

We have done a self-consistent calculation of the electronic structure and magneto-transport properties of a modulationdoped DMH. Our results not only reproduce the experimental findings, but also justify the phenomenological model used in Ref. 6. Since the quantum well is very deep, the main effect of the Hartree contribution is to increase the subband energy by $\sim 57 \mathrm{meV}$, leaving the subband structure almost unchanged.

Note that the value of the fitting parameter $x_{p}=0.093$ is greater than the nominal one $x_{p}=0.0625$. A possible explanation for this discrepancy is that we have neglected the contribution of the exchange-correlation energy of the $2 \mathrm{D}$ electron gas. Its inclusion may enhance the spin splitting [15] thus allowing the use of a smaller value for $x_{p}$, closer to the experimental one [16].

It is worth mentioning that neither the experimental nor our theoretical results show any sign of an itinerant ferromagnetic phase transition [17] at the Landau level crossings. Reference 7 suggests that the effects observed by Knobel $e t$ al. [6] take place in a regime above a critical temperature $T_{c}$. Here we note, in addition, that $B_{c}=3.2 \mathrm{~T}$ corresponds to a filling factor of 3.6, which deviates from the integer filling factor favoring quantum Hall ferromagnetism [18]. The inclusion of exchange and correlation effects should allow us to study the possibility of quantum Hall ferromagnetic states in these magnetic heterostructures [16].

\section{Acknowledgments}

This work was supported by FAPESP (Brazil). HJPF acknowledges Klaus Capelle for useful discussions and R. Knobel for providing additional details on the samples used in Ref.[6].

\section{References}

[1] S. A. Crooker, D. A. Tulchinsky, J. Levy, D. D. Awschalom, R. Garcia, and N. Samarth, Phys. Rev. Lett. 75, 505 (1995).

[2] D. D. Awschalom and N. Samarth, J. Magn. Magn. Mater. 200, 130 (1999).

[3] J. K. Furdyna, J. Appl. Phys. 64, R29 (1988).

[4] I. P. Smorchkova, N. Samarth, J. M. Kikkawa, and D. D. Awschalom, Phys. Rev. Lett. 78, 3571 (1997).

[5] I. P. Smorchkova and N. Samarth, Appl. Phys. Lett. 69, 1640 (1996).

[6] R. Knobel, N. Samarth, J. G. E. Harris, and D. D. Awschalom, Phys. Rev. B 65, 235327 (2002).

[7] J. Jaroszynski, T. Andrearczyk, G. Karczewski, J. Wróbel, T. Wojtowicz, E. Papis, E. Kamińska, A. Piotrowska, D. Popović, and T. Dietl, Phys. Rev. Lett. 89, 266802 (2002).

[8] F. Liaci, P. Bigenwald, O. Briot, B. Gil, N. Briot, T. Cloitre, and R. L. Aulombard, Phys. Rev. B 51, 4699 (1995).

[9] J. C. Egues and J. W. Wilkins, Phys. Rev. B 58, R16012 (1998).

[10] J. A. Gaj, R. Planel, and F. Fishman, Solid State Commun. 29, 435 (1979).

[11] J. A. Gaj, W. Grieshaber, C. Bodin-Deshayes, J. Cibert, G. Feuillet, Y. M. d'Aubigné, and A. Wasiela, Phys. Rev. B 50, 5512 (1994); H. J. P. Freire and J. C. Egues (unpublished).

[12] Since $x_{p}$ is very small the effective Mn concentration is rather insensitive to the actual profile so that $x=x_{p}\left(1-x_{p}\right)^{4}$ is a good first approximation to it. We actually use a segregation profile with characteristic length of $5 \AA$ [11]. A diffusion profile gives similar results.

[13] R. G. Knobel, Ph.D. thesis, Pennsylvania State University, 2000.

[14] T. Ando and Y. Uemura, J. Phys. Soc. Japan 36, 959 (1974).

[15] H. J. P. Freire and J. C. Egues, Braz. J. Phys. 32, 327 (2002); 33, 166(E) (2003).

[16] A similar calculation including exchange and correlation effects will be presented elsewhere.

[17] G. F. Giuliani and J. J. Quinn, Phys. Rev. B 31, 6228 (1985); S. M. Girvin, Phys. Today 53, 39 (2000).

[18] T. Jungwirth and A. H. MacDonald, Phys. Rev. B 63, 035305 (2000). 